\title{
Hn-PERP: Hop by Hop-Power-Efficient Routing Protocol over Underwater Wireless Sensor Networks
}

\author{
Tareq Krishan ${ }^{1}$, Rami S. Alkhawaldeh ${ }^{1}$, Saed Khawaldeh ${ }^{2}$, Bilal Al-Ahmad ${ }^{1} \&$ Adnan Al Smadi ${ }^{3}$ \\ ${ }^{1}$ Department of Computer Information Systems, The University of Jordan-Aqaba, 77110, Jordan \\ ${ }^{2}$ Erasmus+ Joint Master Program in Medical Imaging and Applications; University of Burgundy (France), \\ University of Cassino (Italy) and University of Girona (Spain), Sensor Informatics and Medical Technology \\ Group; Department of Electrical Engineering and Automation, Aalto University (Finland) \\ ${ }^{3}$ Department of Electronics Engineering, Yarmouk University, Irbid, Jordan \\ Correspondence: Tareq Krishan, Department of Computer Information Systems, The University of Jordan-Aqaba, \\ 77110, Jordan. E-mail: t.krishan@ju.edu.jo or tareq_krishan@yahoo.com
}

Received: March 5, 2018 Accepted: October 13, 2018 Online Published: October 29, 2018

doi:10.5539/mas.v12n11p186 URL: https://doi.org/10.5539/mas.v12n11p186

\begin{abstract}
Underwater wireless sensor networks (UWSN) have recently been proposed as a way to monitor and explore the water depths' environments. Efficiently delivering the data is still a challenging problem in these networks because of the weaknesses in the acoustic transmission. To tackle such a problem, we propose a novel algorithm provides controlling mechanisms for critical long-term data forwarding underwater sensor networks, called Hop by Hop Power-Efficient Routing Protocol (Hn-PERP). The proposed Hn-PERP is a centralized full-control model that enhances the network's throughput and energy efficiency by a set of solutions depend on power monitoring in UWSN nodes. In particular, the model provides a guaranteed mechanism for scheduling and processing data transmission based on number of nodes, hops between the nodes, energy level and congestion within each node to minimize energy levels or power consumption by avoiding disconnected probability for any node, which in turn maximizing the network lifetime. Simulation results show that our proposed model is consistent with energy level and congestion, and is more accurate for enabling routing and data transmission. Therefore, the data packet delivery ratio and overall throughput also achieves robust scenarios of very sparse or/and weak networks, to keep on Performance stability in UWSN via adjusting hop-by-hop delay and energy consumption during packages delivery.
\end{abstract}

Keywords: Acoustic communication, Energy consumption, Smart routing protocols, UWSN MAC, Underwater Mobility

\section{Introduction}

In recent decades, UWSNs have emerged as an infrastructure framework to explore environments at specific water depths such as gathering relevant data and monitoring of lakes, rivers, seas, and oceans (Ghoreyshi et al., 2016). UWSNs provide useful sensing capabilities that can be used for short and long-time minor. The wide sensing fields such as temperature, salinity, current movement, video, image, and chemical sensing lead to coherent understanding of underwater circumstances. Therefore, high density feature allows extensive discovering and exploration of wide underwater areas. The infrastructure of such networks consists of a large number of battery-powered vessels, sensors nodes and a variety of devices as shown in Figure 1. Sensor nodes gather and monitor environmental variables (or data sample) such as water temperature, pressure, conductivity, turbidity, biological information, and pollutants by cooperative multi-hop acoustic communication and then deliver them to the surface nodes (or sinks) (Cou, 2015). UWSNs offer the possibility of reconfigure sensors remotely as logical manner and eliminate the need for physically accessing in underwater sites. When unexpected failure is occurred in any sensor in the network, rapid error detection and remote sensing are conducted as applicable features using $\mathrm{UWSN}_{\mathrm{S}}$. 


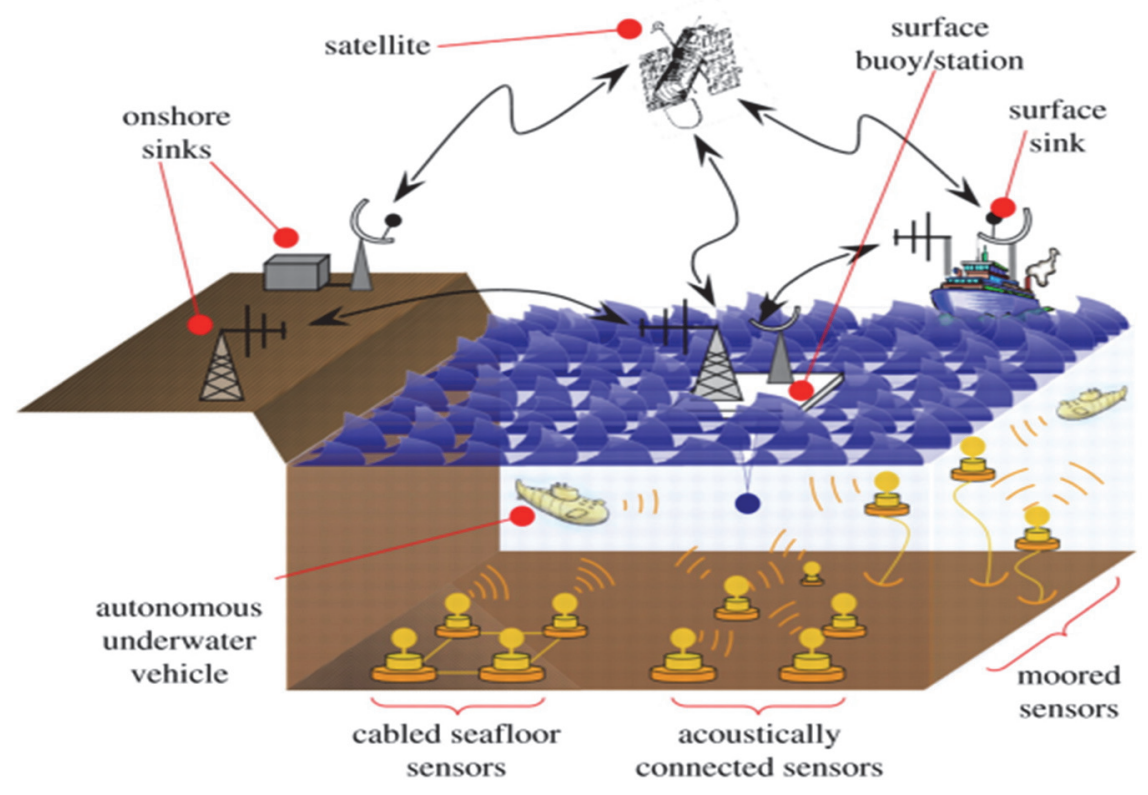

Figure 1. UWSN Infrastructure (Heidemann et al., 2011)

Although these networks have many characteristics as in terrestrial wireless sensor networks, such as numerous nodes and energy issues, they are distinguished from the terrestrial sensor technology in many aspects (Hong et al., 2013). For Instance, regarding communication channels, the RF (Radio Frequency) signaling are not suitable, in underwater environments, due to the long time taken for propagation, large number of low frequencies, and absorption of such signals in water; hence, acoustic signals as a communication medium are exploited to be used in UWSNs (Wahid and Kim, 2010). UWSNs have, however, a broad range of possible applications (Ahmed et al., 2017) such as environmental research, assisted Geographical Information Systems (GISs) navigation, pollution monitoring, real-time control of autonomous underwater vehicles (AUV), and offshore exploration. To make these applications feasible, there is a need for statistical characterization of the acoustic channels (Xiang-ping et al., 2011). In fact, the speed of acoustic signals is five orders of magnitude lesser than radio signals (Umar et al., 2014), which produces long propagation latency and end-to-end delay. Therefore, underwater sensor nodes are energy-constrained and their battery replacement is expensive due to the harsh underwater environment (Wahid et al., 2014). Moreover, the sensors energy level near to the surface is lower than the depth water. Transmission powers in UWSN exceed equivalent free space requirements by at least one order of magnitude. In deep waters, acoustic signals only propagate well at low-frequency signal carriers, which reduce signal bandwidth and transmission rates, thus extending communication time, and then reducing battery life (Uribe and Grote, 2009). These constraints motivate us to propose techniques to enhance network's reliability and throughput in UWSNs, which serve as a challenge. The routing protocols of UWSNs must be calibrated to be more adaptive to the network environment due to achieve reliability by increasing throughput and minimizing delay. Many routing protocols have been proposed for UWSNs, each of these protocols have their own unique characteristics.

In this paper, we present a Power-Efficient Routing Protocol (i.e., Hn-PERP) over renewable paths to enhancing throughput and minimizing delay for critical issues. These issues include congestion and energy level sensors that avoid probabilities of overall performance of downsizing and delay of packet delivery. The optimized patterns of congestion, energy level, and the energy are required for sensor nodes to increase throughput and reduced energy consumption. The idea of our proposed routing protocol developed from using H2-DAB (Hop-by-hop Dynamic Addressing Based) protocol (Ayaz and Abdullah, 2009). H2-DAB interests in a prediction of dynamic addressing for managing mobility nodes movement that is resulted from water current in underwater environments. In this protocol, the water depth conceptually is sliced into different levels from the top to bottom, and thus works only for a relatively short period of data loss. But the nodes at the surface sink consume more energy than the nodes at the bottom because of frequenent uses. For this reason, we devised an algorithm (i.e., Hn-PERP) with which one can obtain more adaptive routing with consideration energy level status in each sensor before transmission routing process. Hence, our contribution is to produce modeling parameters based on 
congestion and energy status. This model depends on full mobility, not on depths or division of layers, to provide high accuracy level in renewable paths. Since it takes optimal routing decision to support stability in the overall throughput synchronously with variations in residual energy within sensors.

The remainder of the paper is organized as follows: Section 2 gives brief reviews of prior research related to present techniques to reduce bandwidth in UWSNs, Section 3 presents our proposed model including Hn-PERP routing protocol and scheduling algorithm. Section 4 explains the experimental parameters and settings as well as discusses the experimental results and evaluation. Finally, conclusions and future work are presented in Section 5 to summaries the results and take away messages.

\section{Related Works}

Many researchers have designed routing protocols based on dynamic topology, but still the research is needed for resolving many problems. Our study focuses on the issues of routing protocol operations regarding to the energy levels and congestions before making decision on routing process within each node.

Several research, however, have been emerged to solve UWSN issues (Ahmed et al., 2017). These researches still needs to investigate and analysis such issues and improve techniques for solving them due to underwater behavior and Environmental conditions. In Random walk (RW) study (Wang and Akyildiz, 2010), any mobile node can move in underwater environment from its current location to a new position in a mathematical approach. This movement depends on direction, speed and range factors without taking into consideration the number of nodes or the number of hops between sensors. Depth-based Protocol (DBR) (Yan et al., 2008) is proposed for the underwater wireless sensor network. In data forwarding mechanism perspective, each sensor node decides a decision on its own depth and the depth of the previous successive sender node, but DBR manages efficiently the resources regardless full dimensional location information of sensor node. The paper in (Zeng et al., 2017) outlines a distributed algorithm to show the benefits of propagation-delays-based interference alignment (PD-IA) in a multi-hop UWSNs with the following consideration in protocol design (message format, control packet delay and overhead, recovery from a lost packet). A set of studies referred to another aspect regarding to the power efficiency within WSNs. A study in (Wang and Zhou, 2017) showed that memory, power supply and processing capacity of sensor nodes are limited; making it difficult to replace and recharge batteries manually and the nodes' energy can be exhausted easily. Many innovative energy harvesting methods, such as wireless energy harvesting (Mekikis et al., 2014) and wind energy harvesting (Li et al., 2016), can promote the long lifetime of wireless sensor networks. However, how to allocate power and use energy effectively is still a problem. UWSNs require enhancing the data rate under limited channel capacity because of the poor channel characteristics. Also, a mathematical analyzed mechanism is needed to monitor the power consumption at nodes by numerical values (Kwon et al., 2015). Due to gathering data at the sink, the transmission/reception data cumulatively increases as they are become closer to the sink and so rapid increases in power consumption. The sensor nodes consume very small amount of energy and the source of energy at these nodes present a challenge to the system designers. Additionally, low capacity and high propagation delay also contribute to the performance degradation of UWSNs. Hence, energy-efficiency and reliability in MAC and protocols are the major concerns in underwater communication (Zenia et al., 2016). Event-to-sink Reliable Transport (ESRT) protocol presented in (Akan and Akyildiz, 2005) comprises a congestion control mechanism that is reliable and conserves energy. In such protocol, the sink decides the actions to take based on the reports received from sensor nodes during decision interval. Thus, the congestion detection mechanism uses local buffer monitoring in the sensor nodes. Moreover, If the buffer of a sensor node is overflowed because of an extravagant amount of data packets, the Congestion Notification $(\mathrm{CN})$ bit in the header of the packets is set and the sink is notified by this procedure. Herein the sink periodically estimates a new reporting rate, at which each source is supposed to report data, on a reliability measurement, the received $\mathrm{CN}$ bits, and the previous reporting rate.

This centralized mechanism cannot be deployed on UWSNs. Congestion control notification messages sent through multiple hops might be lost due to the high link error probability of these networks. In addition to, as opposed to terrestrial sensor networks, the UWSNs have very large propagation delays. Therefore, the tardy response of Event-to-sink congestion control leads to a higher packet loss if there is congestion. For this reason, it is recommended that intermediate nodes rather than the sink participate to react to local congestion (Domingo, 2013). Temporary clustered based routing (TCBR) is table driven underwater routing protocol. TCBR is works on multi-hop and specially designed for equal energy consumption for entire network (Ahmed et al., 2018) with depending on two types of nodes (ordinary and courier). Communication range reached to 500 meters for better power usage. TCBR hello packet consists four fields (Node ID, Expiry time, Hope ID and MAX hop counts) and Data packet structured by five fields (Source Node ID, next node ID, Packet sequence number, Dest ID and Data field). The study in (Ahmed et al., 2018) also does not present directly the contribution through fields of packets 
in TCBR to make sure accurate routing depending on energy level for each node.

As discussed above there is no mechanism present adaptive dynamic addressing synchronously with the following consideration in each node, which are speed, the number of hops between sensors, the number of sensors, congestion in the nodes, depth for each sensor, noise level in the layer and energy level of each sensor. Therefore, our motivation is to build an adaptive approach to doing transmission depending on a renewable path between sensors, energy level for each one, and congestion status making sure forwarding data is performed in an accurate scheduling. These results keep on the stability of network via reducing delay and increasing throughput by adaptable UWSN scheduling.

\section{Hn-PERP: Our Proposed Model}

This section discusses the proposed model as routing protocol and scheduling algorithms. In order to discuss the problem statement first section explains the problem formulation for routing packets in UWSNs.

\subsection{Problem Formulation}

The problem of transferring data packets under UWSNs is formulated as follows: Assume a set of sensors nodes N. A sensor node $\mathrm{S}$ (Sender) sends a packet along a path in the UWSN network to a receiver sensor node R at surface (or sink) in minimum energy and maximum network lifetime.

Where $E(n ; m)$ is the energy consumed when transfer the packet between the sensor node $n$ and sensor node $m$.

$$
\underset{\operatorname{Path}(S, R)=S, n, n+1, \cdots, R}{\arg \min } \sum_{n=S, m=n+1}^{R} E(n, m) \forall m, n \in \operatorname{Path}(S, R)
$$

Hence we have to reduce the whole energy (summation of energies) between nodes in a path between the sender $S$ and receiver $R$, which in turn maximizes the network lifetime. Hence, in this paper, we propose a protocol adopted from $\mathrm{H} 2-\mathrm{DAB}$ protocol to achieve reducing in energy when sending packets and long network lifetime.

\subsection{Hn-PERP Routing Protocol}

Hop to hop - Power-Efficient Routing Protocol (Hn-PERP) is a novel routing protocol that is presented in this study to achieve more adaptive scheduling based on neighbor count, time, congestion and residual energy as development on H2-DAB mechanism (Ayaz and Abdullah, 2009). The proposed model has a set of advantages include:

- In terms of accuracy, the transmission process will be more accurate from node to node. In particular, every node has a prediction about next hop to forward packet in order to avoid probability of delay and increasing throughput over UWSN synchronously with consideration of congestion and energy level.

- If the accuracy of the system is conducted, cost saving, increasing throughput and minimizing delay approaches being a major finding that accurately achieved by Hn-PERP.

Several modifications on UWSN mathematical models with aspects to make delay and energy must consist with current hops number and congestion status to achieve reducing overall delay and enabling of increasing throughput with power consumption over UWSNs. Therefore, the first step is to achieve an adaptive addressing to the scheduling of paths process. Hence, hello and data packets that are provided in H2-DAB must be processed as shown in Figure 2 to be suitable for our future-oriented on introducing an adaptive methodology to the UWSNs.

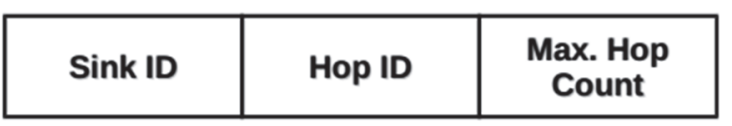

Figure 2. H2-DAB Hello Packet Frame

Sink ID: is a unique ID used to initialize the sinks when they broadcast Hello packets during the first phase. This ID distinguishes the floating nodes when they receive Hello packets from different sinks.

Hop ID: presents the IDs of the number of nodes between two nodes in the system. Left hop number has more priority as a primary route, while as compared to the right hop number that is used as a backup route.

Max. Hop Count: has an initialization value of 10 at broadcasting Hello packet by sink. After receiving the packet, every node decrements the value by one till the tenth node is received to makes the value zero and then 
stops forwarding further to any other nodes. Our proposed protocol Hn-PERP contains new approach to the Hello Packet Frame via H2-DAB Frame as shown in Figure 3 and 4.

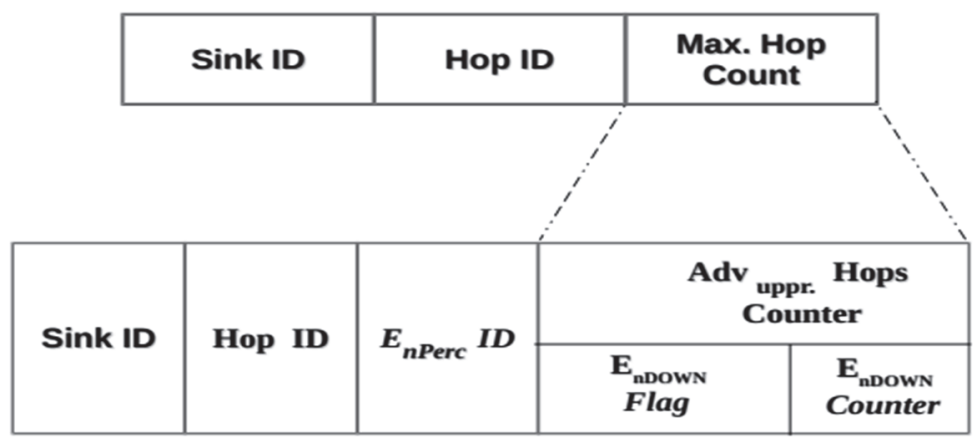

Figure 3. Propose $\mathrm{H}^{n}$-PERP Hello Packet

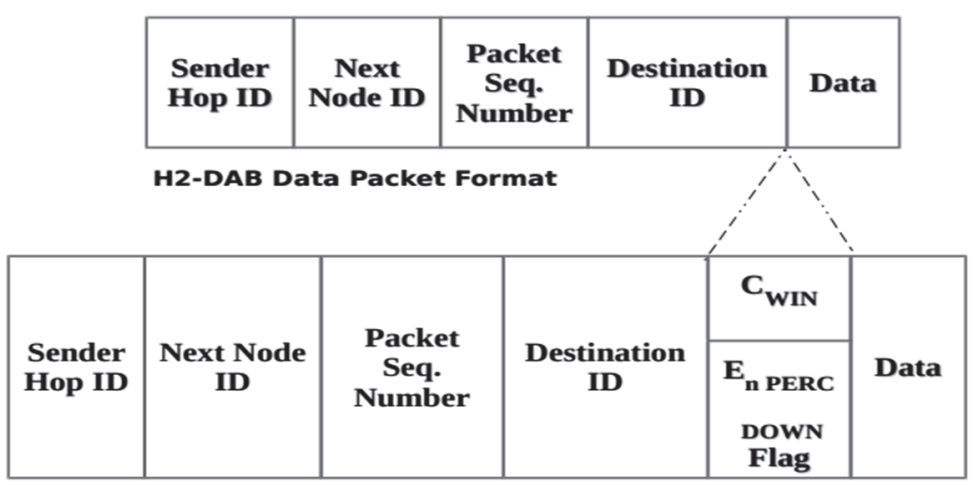

Figure 4. Proposed $\mathrm{H}^{n}$-PERP Data Packet Frame

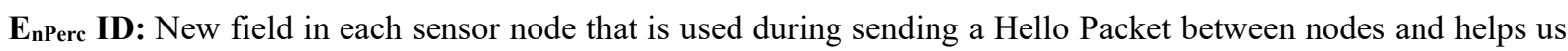
to build scheduling depending on trade-off between nodes by determining residual energy in each node.

Advuppr. Hops Counter: If energy level is more than or equal to $70 \%$, current node is being considered as a next hop.

Endown Flag: it is 0 if energy level in the node $=70 \%$ else the flag is being 1 .

$\mathbf{E}_{\text {nDown }}$ Counter: represents the number of nodes that contain lowest energy in routed path.

Sender hop ID: the Hop ID of current node forwarding the Data Packet, if it's an anchored node then it will use its static Hop ID"100“.

Next Node ID: the unique ID of a node, eligible for next hop among the neighbors, usually a node from the upper layers.

Packet seq. number: a unique number assigned by the source node to the packets.

Destination ID: is a fix value"0", which is the destination ID of all the sinks on the surface, so packets can be delivered to any of the reachable sink.

$\mathbf{C}_{\text {wind: }}$ : Congestion Status in Current node.

EnDown Flag: will be 0 if energy level in the node $=70 \%$ else the flag is 1 .

\subsection{Hn-PERP Scheduling Algorithm}

The scheduling algorithm of Hn-PERP is described in Algorithm 1 and also in Table 1 provides a practical processing to the hop-by-hop trade-off for the nodes during data transmission between suggested sensor 1 and surface sink A over UWSNs. And how making decision depending on our selected parameters in this study to reaches the precision in routing path upon congestion and energy status. 


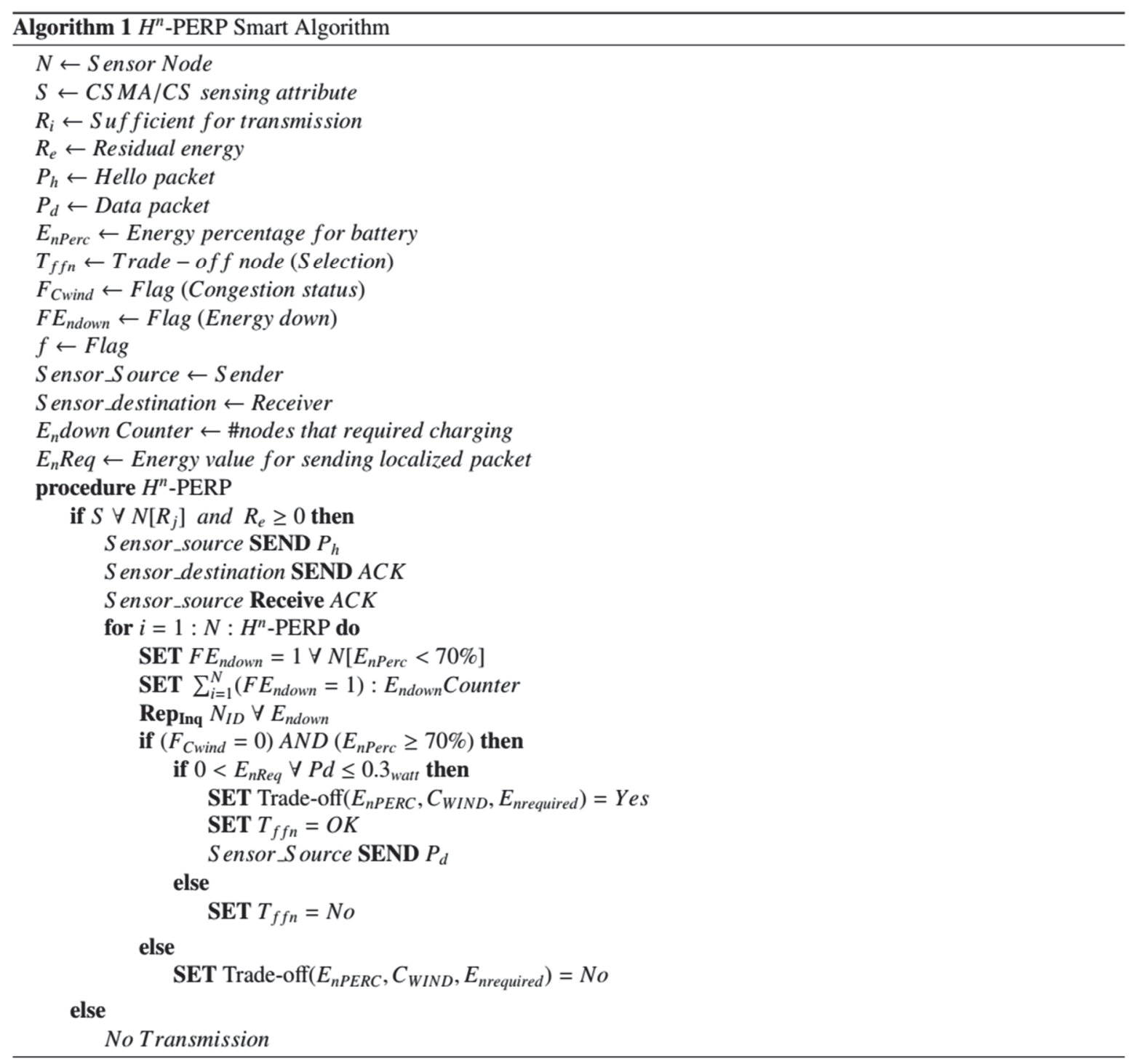

Table 1. $\mathrm{H}^{n}$-PERP Scheduling

\begin{tabular}{|c|c|c|c|c|c|c|c|c|}
\hline & Current node & Hop Number & Reached Node & $\mathbf{C}_{\text {WIND }}$ & $\mathbf{E}_{n P E R C}$ & $\mathbf{E}_{\text {nrequired }}$ & Trade-off $\left(\mathbf{E}_{n P E R C}, \mathbf{C}_{\text {WIND }}, \mathbf{E}_{\text {nrequired }}\right)$ & Trade-off Node \\
\hline \multirow{9}{*}{ 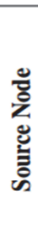 } & 1 & 1 & 6 & 1 & $77 \%$ & 1.51 watt & NO & - \\
\hline & & & 7 & 0 & $74 \%$ & 0.25 watt & Yes & OK \\
\hline & & & 2 & 0 & $84 \%$ & 1.79 watt & NO & - \\
\hline & 7 & 1 & 9 & 1 & $73 \%$ & 1.47 watt & NO & - \\
\hline & & & 13 & 0 & $68 \%$ & 0.27 watt & Yes & Ok \\
\hline & 13 & 1 & 3 & 0 & $59 \%$ & 0.26 watt & Yes & OK \\
\hline & 3 & 1 & $\mathbf{n}$ & $\cdots$ & $\cdots$ & $\cdots$ & $\cdots$ & $\cdots$ \\
\hline & 3 & 1 & $\mathbf{n}$ & $\cdots$ & $\cdots$ & $\cdots$ & $\cdots$ & $\cdots$ \\
\hline & 18 & 1 & A & - & - & - & - & - \\
\hline
\end{tabular}

\subsection{Hn-PERP Evaluation}

Generally, the Energy efficiency, throughput, delay, packet delivery ratio (PDR) and reliability are the most common performance metrics used for estimating MAC protocols for UWSNs. The definition of the performance metrics are presented as follows:

Energy efficiency: is defined as the total amount of energy consumed over the total simulation time. These include all the energy spent in transmission, reception, idle and sleep state etc.

Throughput: is defined as the amount of data packets successfully transmitted from sender to receiver over the 
total simulation time $(\mathrm{T})$. We compute throughput as number of bits $(\mathrm{N})$ received successfully by all destinations. Mathematically, throughput can be defined as: N/T.

Delay: end-to-end delay can be defined as the average time taken by a data packet to arrive in the destination, which can be mathematically defined as $\mathrm{D}=$ Sum (arrival time - sending time) / Sum (Number of connections).

Packet Delivery Ratio (PDR): is the ratio of number of delivered data packet to the destination. This illustrates the level of delivered data to the destination. PDR can be mathematically defined as Sum (Number of packet receive) / Sum (Number of packet send).

Reliability: could be defined as guaranteed delivery of transmitted data in UWSNs to intended receiver (s), which also provides notifications to sender.

Hence, our selected metrics in the proposed study focus on the overall throughput in the suggested network and energy percentages within each node over UWSN.

\section{Experimental Results}

\subsection{Experimental Parameters and Settings}

Herein, we discuss the re-formalization of the packets' headers that are presented in (Ayaz and Abdullah, 2009) and consider parameters to be included for building a new adaptive routing mechanism. Hence, in order to achieve that, we have a set of steps as follow:

System architecture: this step defines the architecture that have to be used for building the proposed model which includes:

- Develop H2-DAB Frames to solve our issue and make routing process more adaptive with specific UWSN considerations such as neighbor hops, congestion, and residual energy.

- Build a scheduling for routing paths to make sure that addressing processing supports increasing throughput that is obtained by $\mathrm{H} 2-\mathrm{DAB}$.

- Build a simulation to evaluate the proposed model.

Table 2. Experimental parameters and vaues

\begin{tabular}{|c|c|}
\hline \multicolumn{1}{|c|}{ Parameters } & values \\
\hline Shannel-type & UWSN Acoustic Channel \\
\hline Propagation-Model & BH/SPM OR OPNET \\
\hline Mac-type & IEEE 802.15.4 CSMA/CS \\
\hline Mac-Data-Rate & $30 \mathrm{MB} / \mathrm{s}$ \\
\hline Mac-Bandwidth-Rate & $100 \mathrm{Khz}$ \\
\hline Link-Layer-Type & Adaptive routing \\
\hline Antenna-Model & Acoustic wave \\
\hline Network-Interface-Type & n $/ 1$ Queuing \\
\hline No. of sensors Nodes & 1000 \\
\hline Max-packet-In-Interface-Queue & Drop tail-priority Queue \\
\hline Interface-Queue-type & Var (rp); [H2-DAB or H ${ }^{n}$-PERP] \\
\hline Routing Protocol & 12 hours \\
\hline Simulation time & Up to 0.3 watts \\
\hline Energy percentage & Assigned as in Step One \\
\hline Required energy & Defined Previously \\
\hline & Step Two \\
\hline Nodes-Configuration & Randomly \\
\hline Initial distribution for the nodes & For the receiver \\
\hline Sensor Nodes Motion & Step Three \\
\hline &
\end{tabular}


Pre-processing step: This represents the characteristics of our UWSN environment. The simulation setup includes the parameters and values that are selected depend on experimental tests. We randomly use varying numbers of nodes ranging from 5 to 50 in 3D region of size $1500 \mathrm{~m}$ X $1500 \mathrm{~m}$. Sensor nodes have a transmission range of $200 \mathrm{~m}$, the data rate of $2 \mathrm{MB} / \mathrm{sec}$, and use the CSMA MAC protocol. The size of the packet payload is 512 bits. The values of energy consumption are not required, which are up to 0.3 watts for transmission and reception. This configuration is used throughout, which summarized in Table 2.

Design: In this step, we present the design of Hn-PERP, A practical Adaptive routing Architecture schema for UWSN as shown in Figure 6. Hn-PERP transparently addresses trade-off events in a smart sensing and routing while it allows flexible control sensors nodes.

\subsection{Experimental Results and Evaluation}

We evaluate the performance of Hn-PERP using the standard NS2 simulation. In our simulations, we considered 50 sensor nodes deployed within $1500 \mathrm{~m}$ X $1500 \mathrm{~m}$ X $1500 \mathrm{~m}$ with a packet size of 512 bit and Max-packet-In-Interface-Queue up to 1000. Our performance metrics are throughput and number of nodes in the overall proposed network to evaluate the efficiency of Hn-PERP protocol.

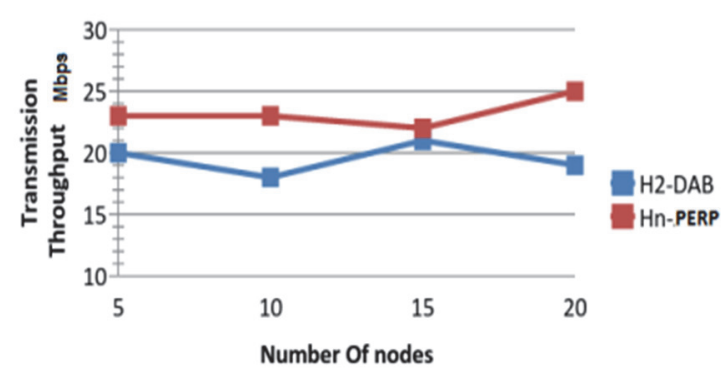

(a) 20 Nodes

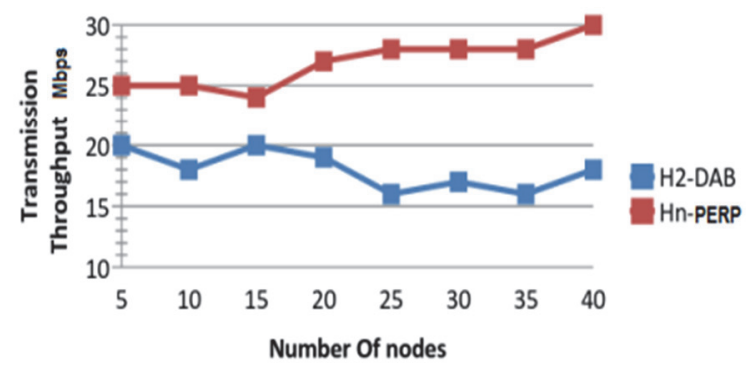

(c) 40 Nodes

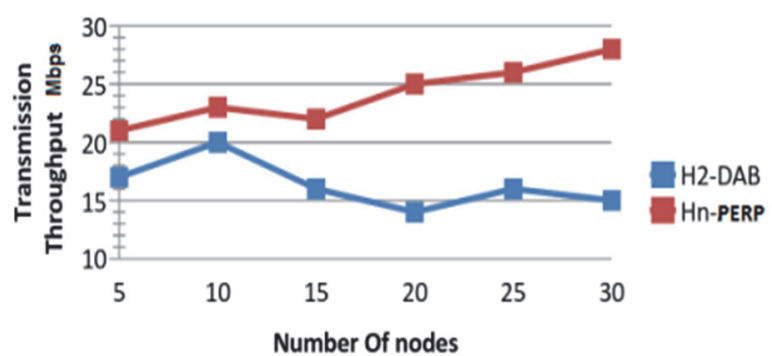

(b) 30 Nodes

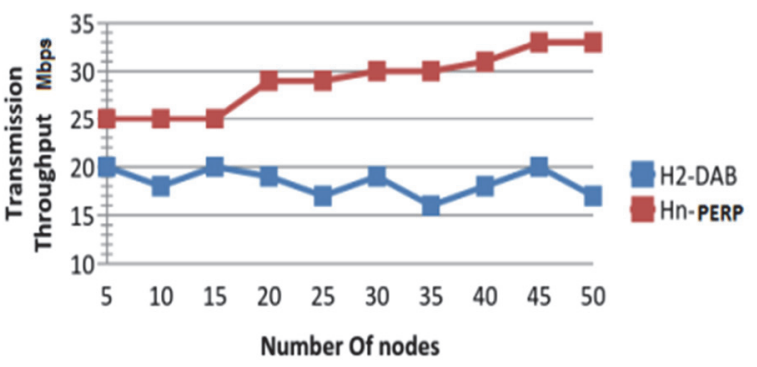

(d) 50 Nodes

Figure 5. $\mathrm{H}^{n}$-PERP Throughput

\begin{tabular}{|c|c|c|}
\hline \multicolumn{3}{|c|}{ Hello Packet Frame } \\
\hline \multicolumn{3}{|c|}{ Data-packet Frame } \\
\hline $\begin{array}{c}\text { State Scheduling } \\
\text { based on } \\
\text { neighbor count, } \\
\text { congestion status, } \\
\text { residual energy }\end{array}$ & $\begin{array}{c}\text { Mathematical model } \\
\text { regarding to the } \\
\text { minimizing UWSN } \\
\text { delay }\end{array}$ & $\begin{array}{c}\text { Mathematical } \\
\text { model regarding to } \\
\text { the overall } \\
\text { power } \\
\text { consumption } \\
\text { within UWSN }\end{array}$ \\
\hline
\end{tabular}

Figure 6. $\mathrm{H}^{n}$-PERP Architecture Schema

As shown in Figure 5, Hn-PERP results show that the data transmission rate increasing synchronously with the increase in the number of nodes, changing in energy levels, differentiation in a residual energy and the different 
statuses of congestion in a distributed sensor nodes. This makes our proposed routing algorithm more adaptive and precision in different perceptions over UWSN depending on Hn-PERP mechanism. In more detail, the data transmission rate reaches to $33 \mathrm{Kbps}$ with 50 nodes. Our focus here is to increase data bit rate level with a more precise energy level and congestion statues. Therefore, enabling the data transmission to be more stable with overall considerations as power consumption and congestion. On the other hand, H2-DAB present decrements in data ratio during increase with a number of nodes which ranges between 15-20 Kbps through 30 nodes.

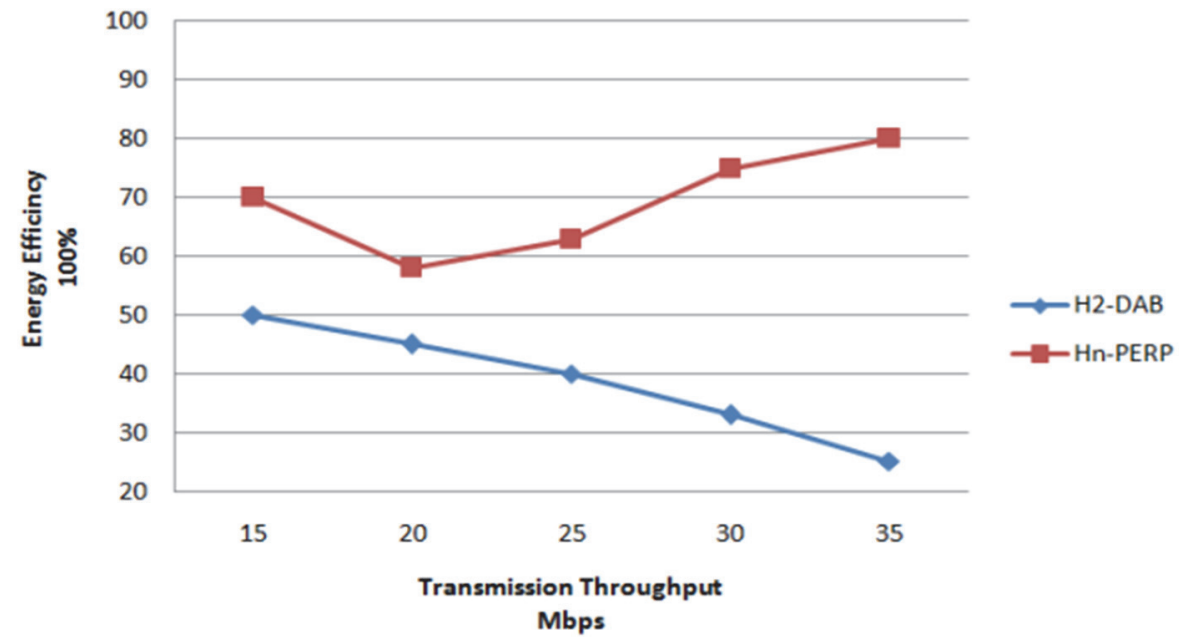

Figure 7. $\mathrm{H}^{n}$-PERP Performance

The energy efficiency regarding our proposed method is shown in Figure 7. As shown, the low level of energy efficiency in H2-DAB is synchronous with increasing productivity on the network occurred by increased probability of delay due to inefficient routing processes. This occurs due to the energy levels that are very low so increasing the opportunities of disconnect in data transmission between nodes. On the other hand, significant stability in the energy efficiency of Hn-PERP protocol reflects to the minimum delay opportunities on the network due to our proposed routing mechanism based on multiple requirements within each node such as energy level, thus, avoiding the contact with low energy nodes.

\section{Conclusions and Future Works}

Most of the UWSN have a risk in energy level especially if we argue about specific applications related to the multimedia data flow in different GIS and environmental usages, which need to be optimized in the packets delivery without being affected by the low level of energy within the sensor nodes. Transmission over these networks has to react to the dynamic change in the nodes as residual energy and congestion. So, adaptability using energy-efficient can handle the failure of single or multiple nodes. In this paper, we proposed Hn-PERP as smart mechanism which was determined to be suitable for UWSNs. This paper discussed the influence of different parameters involved in presented routing scheduling as: hops count, energy level, energy required to packet forwarding and congestion to improve the performance according to the changing trend of UWSNs, design of adaptive addressing based routing algorithm which include stability of power consumption and Increasing of throughput over network as a promising network for the future. Our results showed the stability of the network performance in synchronizing with the increase in the number of nodes and the variation differencein the levels of energy in the contract that did not affect the productivity of the network.

In the future, we are planning to apply some modifications to a specific mathematical model which applied on UWSN propagation delay to consist with accurate throughput and avoidance maximizing delay. In addition, we will extend conducting the experimental simulation to compare the results with other mathematical models regarding the energy requested value depending on Packet size and examine the minimize of overall delay over underwater sensor networks and continue with improvements on suggested equations to reach needed optimization.

\section{References}

Ahmed, M., Salleh, M., \& Channa, M. (2017). Routing protocols based on node mobility for underwater wireless sensor network (uwsn): A survey. Journal of Network and Computer Applications, 78, $242-252$. https://doi.org/10.1016/j.jnca.2016.10.022 
Ahmed, M., Salleh, M., \& Channa, M. (2018). Routing protocols based on protocol operations for underwater wireless sensor network: A survey. Egyptian Informatics Journal, 19(1), 57-62. https://doi.org/10.1016/j.eij.2017.07.001.

Akan, O. B., \& Akyildiz, I. F. (2005). Event-to-sink reliable transport in wireless sensor networks. IEEE/ACM Transactions on Networking, 13(5), 1003-1016.https://doi.org/10.1109/TNET.2005.857076

Ayaz, M., \& Abdullah, A. (2009). Hop-by-hop dynamic addressing based (h2-dab) routing protocol for underwater wireless sensor networks. In Proceedings of the 2009 International Conference on Information and Multimedia Technology, ICIMT '09, pages 436-441, Washington, DC, USA. IEEE Computer Society. https://doi.org/10.1109/ICIMT.2009.70

Coutinho, R. W., Boukerche, A., Vieira, L. F., \& Loureiro, A. A. (2015). A novel void node recovery paradigm for long-term underwater sensor networks. Ad Hoc Networks, 34,144-156. https://doi.org/10.1016/j.adhoc.2015.01.012

Domingo, M. C. (2013). Marine communities based congestion control in underwater wireless sensor networks. Information Sciences, 228, 203-221. https://doi.org/10.1016/j.ins.2012.11.011

Ghoreyshi, S. M., Shahrabi, A., \& Boutaleb, T. (2016). A novel cooperative opportunistic routing scheme for underwater sensor networks. Sensors, 16(3). https://doi.org/10.3390/s16030297

Heidemann, J., Stojanovic, M., \& Zorzi, M. (2011). Underwater sensor networks: Applications, advances and challenges. Philosophical Transactions of the Royal Society of London A: Mathematical, Physical and Engineering Sciences, 370(1958), 158-175. https://doi.org/10.1098/rsta.2011.0214

Hong, L., Hong, F., Yang, B., \& Guo, Z. (2013). Ross: Receiver oriented sleep scheduling for underwater sensor networks. In Proceedings of the Eighth ACM International Conference on Underwater Networks and Systems, WUWNet '13, pages 41-48, New York, NY, USA. ACM. https://doi.org/10.1145/2532378.2532396

Kwon, J. K., Seo, B. M., Yun, K., \& Cho, H. S. (2015). Time-efficient high-rate data flooding in one-dimensional acoustic underwater sensor networks. Sensors, 15(11), 27671-27691. https://doi.org/10.3390/s151127671

Li, Z., Zhou, G., Zhu, Z., \& Li, W. (2016). A study on the power generation capacity of piezoelectric energy harvesters with different fixation modes and adjustment methods. Energies, 9(2), 98. https://doi.org/10.3390/en9020098

Mekikis, P. V., Lalos, A. S., Antonopoulos, A., Alonso, L., \& Verikoukis, C. (2014). Wireless energy harvesting in two-way network coded cooperative communications: A stochastic approach for large scale networks. IEEE Communications Letters, 18(6), 1011-1014. https://doi.org/10.1109/LCOMM.2014.2320926.

Umar, A., Hasnat, M., Behzad, M., Baseer, I., Khan, Z., Qasim, U., \& Javaid, N. (2014). On enhancing network reliability and throughput for critical-range based applications in uwsns. Procedia Computer Science, 34, 196-203. https://doi.org/10.1016/j.procs.2014.07.089

Uribe, C., \& Grote, W. (2009). Radio communication model for underwater wsn. In Proceedings of the 3rd International Conference on New Technologies, Mobility and Security, NTMS'09, pages 147-151, Piscataway, NJ, USA. IEEE Press.

Wahid, A., \& Kim, D. (2010). Analyzing routing protocols for underwater wireless sensor networks. IJCNIS, $2(3)$.

Wahid, A., Lee, S., \& Kim, D. (2014). A reliable and energy-efficient routing protocol for underwater wireless sensor networks. International Journal of Communication Systems, 27(10), 2048-2062. https://doi.org/10.1002/dac.2455

Wang, H., \& Zhou, G. (2017). Power allocation based on data classification in wireless sensor networks. Sensors, 17(5). https://doi.org/10.3390/s17051107.

Wang, P., \& Akyildiz, I. F. (2010). Effects of different mobility models on traffic patterns in wireless sensor networks. In 2010 IEEE Global Telecommunications Conference GLOBECOM 2010, pages 1-5. https://doi.org/10.1109/GLOCOM.2010.5684190

Xiang-ping, G., Yan, Y., \& Rong-lin, H. (2011). Analyzing the performance of channel in underwater wireless sensor networks (uwsn). Procedia Engineering, 15, 95-99. https://doi.org/10.1016/j.proeng.2011.08.020

Yan, H., Shi, Z. J., \& Cui, J. H. (2008). DBR: Depth-based routing for underwater sensor networks, p. 72-86. 
Springer Berlin Heidelberg, Berlin, Heidelberg.

Zeng, H., Hou, Y. T., Shi, Y., Lou, W., Kompella, S., \& Midkiff, S. F. (2017). A distributed scheduling algorithm for underwater acoustic networks with large propagation delays. IEEE Transactions on Communications, 65(3), 1131-1145. https://doi.org/10.1109/TCOMM.2017.2647940.

Zenia, N. Z., Aseeri, M., Ahmed, M. R., Chowdhury, Z. I., \& Kaiser, M. S. (2016). Energy-efficiency and reliability in fMACg and routing protocols for underwater wireless sensor network: A survey. Journal of Network and Computer Applications, 71, 72-85.

\section{Copyrights}

Copyright for this article is retained by the author(s), with first publication rights granted to the journal.

This is an open-access article distributed under the terms and conditions of the Creative Commons Attribution license (http://creativecommons.org/licenses/by/4.0/). 